\title{
Multi-objective Offloading Decision Based on Combination Weighting Method for Multi-access Edge Computing
}

\author{
Dan Ye ${ }^{1,2}$, Xiaogang Wang ${ }^{1,2, *}$, Jin Hou ${ }^{1,2}$ \\ ${ }^{1}$ School of Automation and Information Engineering, Sichuan University of Science and Engineering, Yibin, China \\ ${ }^{2}$ Artificial Intelligence Key Laboratory of Sichuan Province, Sichuan University of Science and Engineering, Yibin, China
}

Email address:

Yedan_ydd@163.com (Dan Ye),wxg_zf@163.com (Xiaogang Wang)

${ }^{*}$ Corresponding author

\section{To cite this article:}

Dan Ye, Xiaogang Wang, Jin Hou. Multi-objective Offloading Decision Based on Combination Weighting Method for Multi-access Edge Computing. Internet of Things and Cloud Computing. Vol. 9, No. 3, 2021, pp. 21-26. doi: 10.11648/j.iotcc.20210903.11

Received: December 8, 2021; Accepted: December 21, 2021; Published: December 29, 2021

\begin{abstract}
Generally, in the edge computing scenario, edge devices can offload tasks to the edge servers to reduce device energy consumption and task execution delay. It is necessary to find an offloading strategy which can balance and minimize the task execution delay and device energy consumption. This is usually classified as a multi-objective problem. It is a common method to get the Pareto optimal solution set by using multi-objective optimization algorithm. However, there is a problem about how to find out the eclectic optimal solution that can embody the user's subjective consciousness and meet the objective information of Pareto optimal solution set. This paper solved this problem by combining subjective and objective combination weighting method. First, the subjective weight matrix which reflects the user's subjective consciousness is obtained by analytic hierarchy process. Then, the objective weight matrix which can embody the objective information of the index is obtained through the entropy method. Finally, the combination weight matrix is obtained by subjective and objective weighting method. After comprehensively evaluating the Pareto optimal solution set, the solution with the minimum comprehensive evaluation value is regarded as the Pareto compromise optimal solution. In this paper, the combination weighting method is applied to multi-access edge computing scenario and verify its feasibility in this scenario.
\end{abstract}

Keywords: Edge Computing, Combination Weighting Method, Multi-objective Optimization

\section{Introduction}

In 2007 , the concept of cloud computing was formally put forward, and mobile cloud computing based on cloud computing concept has been widely used [1]. Mobile cloud computing (MCC) can access powerful remote centralized cloud servers through core networks and the internet to get powerful computing and storage resources [2-3]. However, with the development of 5G technology and the popularization of Internet of things technology, a large number of terminals have accessed the core network, and a large amount of data overwhelmed the core network [4-5]. In addition, some new strong delay technologies have begun to improve the requirements of network transmission delay. MCC technology cannot meet the growing demand for technology [6-7].

In 2014, mobile edge computing was formally proposed by the European Telecommunications Standards Institute (ETSI) [8]. In 2017, ETSI extended the concept of MEC to other access networks (such as Wi-Fi, vehicle network and even fixed network) and renamed it as Multi-access Edge Computing [9]. The purpose of MEC technology is to provide users with computing power and IT service environment closer to the user's network edge.

Computing offloading is a key technology in MEC. It refers to that the terminal equipment unloads some or all computing tasks to the edge computing environment for processing through Internet technology [10]. A lot of researchers have studied the offloading technology of MEC. In the research process, task execution delay and equipment energy consumption are mainly used as performance indicators. Some scholars have studied the single performance index, while others have studied the 
offloading technology that takes performance execution time delay and device energy consumption as performance indicators. This is actually a multi-objective optimization problem. In order to solve such problems, scholars usually combine MEC offloading problem with multi-objective optimization algorithm. Cui et al. proposed an improved algorithm based on NSGA II for the task execution delay and device energy consumption in the Internet of things scenario [11]. The algorithm can obtain a series of uniformly distributed Pareto optimal solutions.

However, how to find out the eclectic optimal solution to meet the needs of users from the Pareto optimal solution set is a problem to be explored. It is meaningless to use the exponential function or logarithmic function to standardize the solution set. Therefore, effective index weight need be determined. This paper studies the combination weighting method combining subjective weighting method and objective weighting method. It is able to find out the eclectic optimal solution to meet the needs of users from the Pareto optimal solution set.

\section{Related Works}

\subsection{Multi-objective Optimization}

In real situations, many problems are made up of multiple objectives that are conflicting and affecting. These objectives cannot be achieved at the same time. We usually try to make these objectives reach the best state within a certain range, this is called multi-objective optimization [12].

Generally, multi-objective problems can be described as:

$$
\begin{gathered}
\min f(x)=\left(f_{1}(x), \ldots, f_{n}(x)\right)^{T} \\
\text { s.t. } g_{i}(x) \geq 0, i \in I \\
h_{j}(x)=0, j \in E
\end{gathered}
$$

where $f_{i}(x),\{i=1,2,3 \ldots, n\}$ is the objective function, $g_{i}(x)$ and $h_{j}(x)$ are constraint functions.

There are some definitions of multiple objectives that need to be elaborated:

Definition 1: Pareto dominate

Consider two decision vectors $a, b \in X$. If and only if: $\left\{\forall \mathrm{i} \in\{1,2, \ldots, \mathrm{n}\} \mathrm{f}_{\mathrm{i}}(\mathrm{a}) \leq \mathrm{f}_{\mathrm{i}}(\mathrm{b})\right\} \wedge\left\{\exists \mathrm{j} \in\{1,2, \ldots, \mathrm{n}\} \mathrm{f}_{\mathrm{j}}(\mathrm{a})<\mathrm{f}_{\mathrm{j}}(\mathrm{b})\right\}$, we think $a$ Pareto Dominate $b$, called $a>b$.

Definition 2: Pareto optimal solution

If there is a decision vector in space that is not dominated by any other vector. This decision vector is the Pareto optimal solution. The set composed of all Pareto optimal solutions is called Pareto optimal solution set.

Definition 3: Pareto front

If there are no other decision variables that can dominate a decision variable, the decision variable is called a non-dominated solution.

Generally, the non-dominated solution of multi-objective optimization problems is more than one. A set consisting of all non-dominated solutions is called a non-dominated set. The objective functions corresponding to all non-dominated solutions constitute the non-dominated optimal objective domain, which is called Pareto front.

As shown in Figure 1, four non-dominated points of $A B C D$ constitute Pareto front. $A B C D$ is the dominant point of point $E F$. There is no dominant relationship between $A B C D$.

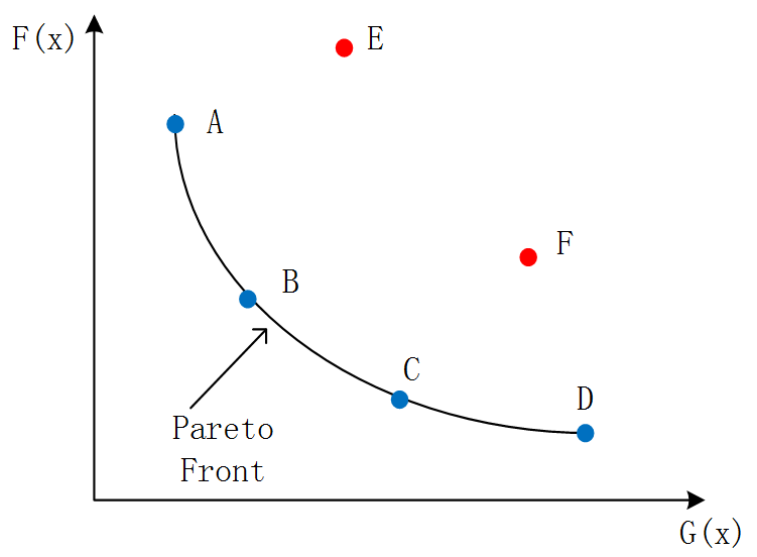

Figure 1. Pareto Front.

\subsection{Weighting Method}

The weighting method can be roughly divided into three categories: subjective weighting method, objective weighting method and integrated weighting method.

The subjective weighting method is based on the subjective experience and judgment of decision-makers, and uses certain specific rules to measure the weights of indicators. It mainly depends on decision-makers' subjective judgments. The commonly used methods are: serial ratio method, expert consultation method, analytic hierarchy process, etc. [13]

Objective weighting method is a method to determine the weight with a specific law according to the objective information provided by the decision matrix. It mainly depends on the degree of connection between indicators, the amount of information provided by each indicator, and the impact on other indicators. The common methods are: principal component analysis, improved ideal solution, entropy method and so on. [14]

However, in the process of weighting, the objective weighting method relies on the objective information of indicators, and does not take into account the subjective needs of decision makers. This is unreasonable in the actual weighting. In addition, although the subjective weighting method can consider and rely on the subjective needs of decision makers, it does not consider the objective relationship between indicators. Therefore, the subjective weighting method is also impractical.

Combination weighting method is a method that integrates subjective and objective weighting methods to determine the weight situation [15]. It organically combines subjective weighting method and objective weighting method to enhance their complementarity. and makes the weight distribution pay attention to the objective information of indicators while considering the subjective needs of decision makers. 


\section{Decision Making Method Based on Combination Weighting Method}

In the research of MEC's offloading strategy, there are many researches on joint offloading strategy for finding the execution delay and energy consumption of multi-access computing tasks in the Internet of things scenarios. Researchers usually combine it with multi-objective optimization algorithms, such as NSAG-ii, SPEA2 and so on. Eventually, the Pareto optimal set that meets the requirements of the current scenario would be obtained. However, some studies focus on the robustness of the Pareto front. There is a lack of systematic strategy to find out the compromise optimal solution to meet the needs of users from the Pareto optimal set. It is meaningless to simply use natural functions or logarithmic functions. Considered with the combination weighting method, this paper selects the compromise optimal solution from the Pareto optimal set with a more scientific and effective method. Based on the characteristics of the combined weighting method, it considers the subjective needs of decision makers and the objective information of the data at the same time.

\subsection{Normalization}

In the MEC scenario, the Pareto optimal set obtained by multi-objective optimization algorithm is different in the type and dimension of different target sets. Before weighting, the content of the target set needs to be normalized. Usually, the optimization target in the MEC scenario is to find the minimum value that can satisfy the objective function. Therefore, here we choose a normative method for small indicators, which can be given by:

$$
\mathrm{Z}_{\mathrm{ij}}=\frac{\mathrm{Max}_{\mathrm{j}}}{\mathrm{x}_{\mathrm{ij}}}
$$

Here, $\operatorname{Max}_{\mathrm{j}}=\max _{\mathrm{i} \in \mathrm{N}} \mathrm{x}_{\mathrm{ij}}, \mathrm{N}$ is the corresponding target set of Pareto optimal set.

From the above summary, for a Pareto optimization solution set with $\mathrm{M}$ optimization goals and $\mathrm{N}$ optimal solutions, We can get a $\mathrm{n} \times \mathrm{m}$ matrix $\mathrm{A}=\left(\mathrm{a}_{\mathrm{ij}}\right)_{\mathrm{n} \times \mathrm{m}}$ by normalization.

\subsection{Determining the Subjective Weight}

In the combination weighting method, decision-makers need to combine various subjective weighting methods when dealing with more complicated decisions. However, in the MEC unloading decision, the types of indicators selected are usually limited. Therefore, this paper chooses the analytic hierarchy process of subjective decision method to get the subjective weight matrix $\mathrm{W}_{\mu}=\left[\mathrm{w}_{\mu 1}, \mathrm{w}_{\mu 2}, \ldots, \mathrm{w}_{\mu \mathrm{m}}\right]$.

Firstly, we need to establish a decision matrix composed of decision elements. Then, by comparing the decision elements in pairs, the weight of each decision element is obtained. For example, we need to get the subjective weight matrix of decision sets with three decision objectives $O_{1}, O_{2}, O_{3}$. The decision matrix of the decision set is:

$$
\mathrm{O}=\left[\begin{array}{ccc}
1 & 1 / 3 & 1 / 5 \\
3 & 1 & 1 / 3 \\
5 & 3 & 1
\end{array}\right]
$$

Here, policymakers consider that $\mathrm{O}_{3}$ is the most important, $\mathrm{O}_{2}$ is more important, and $O_{1}$ is the least important. The importance of decision itself is the same for decision making itself, so the diagonal value of the matrix should be 1 . The importance of $O_{2}$ versus $O_{1}$ and the importance of $O_{1}$ to $O_{2}$ are reciprocal, and so on. Then, we use the arithmetic mean method to estimate the weight value:

$$
\mathrm{w}_{\mu \mathrm{i}}=\frac{1}{\mathrm{n}} \sum_{\mathrm{j}=1}^{\mathrm{n}} \frac{\mathrm{o}_{\mathrm{ij}}}{\sum_{\mathrm{k}=1}^{\mathrm{n}} \mathrm{o}_{\mathrm{kj}}}
$$

\subsection{Determining the Objective Weight}

In this section, we determine the objective weight matrix $\mathrm{W}_{\mathrm{v}}=\left[\mathrm{w}_{\mathrm{v} 1}, \mathrm{w}_{\mathrm{v} 2}, \ldots, \mathrm{w}_{\mathrm{vm}}\right]$ by entropy method. First, we use the equation (2) to get the normalized matrix $A=\left(a_{i j}\right)_{n \times m}$. Then, calculate the proportion of the $\mathrm{i}$-th optimal solution under the $\mathrm{j}$-th index in the target:

$$
\mathrm{p}_{\mathrm{ij}}=\frac{\mathrm{a}_{\mathrm{ij}}}{\sum_{\mathrm{i}=1}^{\mathrm{n}} \mathrm{a}_{\mathrm{ij}}}
$$

Calculate the entropy of $\mathrm{j}$-th index:

$$
\mathrm{e}_{\mathrm{j}}=-\left(\frac{1}{\ln (\mathrm{n})}\right) \sum_{\mathrm{i}=1}^{\mathrm{n}} \mathrm{p}_{\mathrm{ij}} \ln \left(\mathrm{p}_{\mathrm{ij}}\right)
$$

Calculate information entropy redundancy:

$$
\mathrm{d}_{\mathrm{j}}=1-\mathrm{e}_{\mathrm{j}}
$$

Calculate the weight of each index:

$$
\mathrm{W}_{\mathrm{vj}}=\frac{\mathrm{d}_{\mathrm{j}}}{\sum_{\mathrm{j}=1}^{\mathrm{m}} \mathrm{d}_{\mathrm{j}}}
$$

\subsection{Determining Combination Weights}

The integrated combination weight can be expressed as: $\mathrm{W}=\left(\mathrm{w}_{1}, \mathrm{w}_{2}, \ldots, \mathrm{w}_{\mathrm{m}}\right)$. Here, $\mathrm{w}_{\mathrm{j}}$ satisfies the condition of $\sum_{\mathrm{j}=1}^{\mathrm{m}} \mathrm{w}_{\mathrm{j}}=1, \mathrm{w}_{\mathrm{j}} \geq 0$. Finally, the scheme can be comprehensively evaluated according to the weight of integration:

$$
\mathrm{y}_{\mathrm{i}}=\sum_{\mathrm{j}=1}^{\mathrm{m}} \mathrm{w}_{\mathrm{j}} \mathrm{a}_{\mathrm{ij}}
$$

We use distance to describe the deviation of weighting method. The deviation of the evaluation result is expressed by the Euclidean distance between the combined weighted evaluation value and the subjective and objective weighted evaluation value. In order to make the weight of the combination more reasonable, we strive to minimize the total Euclidean distance, because the smaller the better on the deviation of the evaluation results. Applying the least squares method, the combined weights can be calculated:

$$
\left\{\begin{array}{c}
\min \sum_{\mathrm{i}=1}^{\mathrm{n}} \sum_{\mathrm{j}=1}^{\mathrm{m}}\left[\left(\mathrm{w}_{\mathrm{j}}-\mathrm{w}_{\mu \mathrm{j}}\right) \mathrm{a}_{\mathrm{ij}}\right]^{2}+\left[\left(\mathrm{w}_{\mathrm{j}}-\mathrm{w}_{\mathrm{vj}}\right) \mathrm{a}_{\mathrm{ij}}\right]^{2} \\
\text { s.t. } \sum_{\mathrm{j}=1}^{\mathrm{m}} \mathrm{w}_{\mathrm{j}}=1, \mathrm{w}_{\mathrm{j}} \geq 0 .
\end{array}\right.
$$




\section{Example Analysis}

This paper considers an Internet of things scenario with multiple users and multiple MEC servers. The task to be processed by each user are considered independent. The tasks can be offloaded to the MEC server or locally to handle. The MEC scenario is shown in Figure 2.

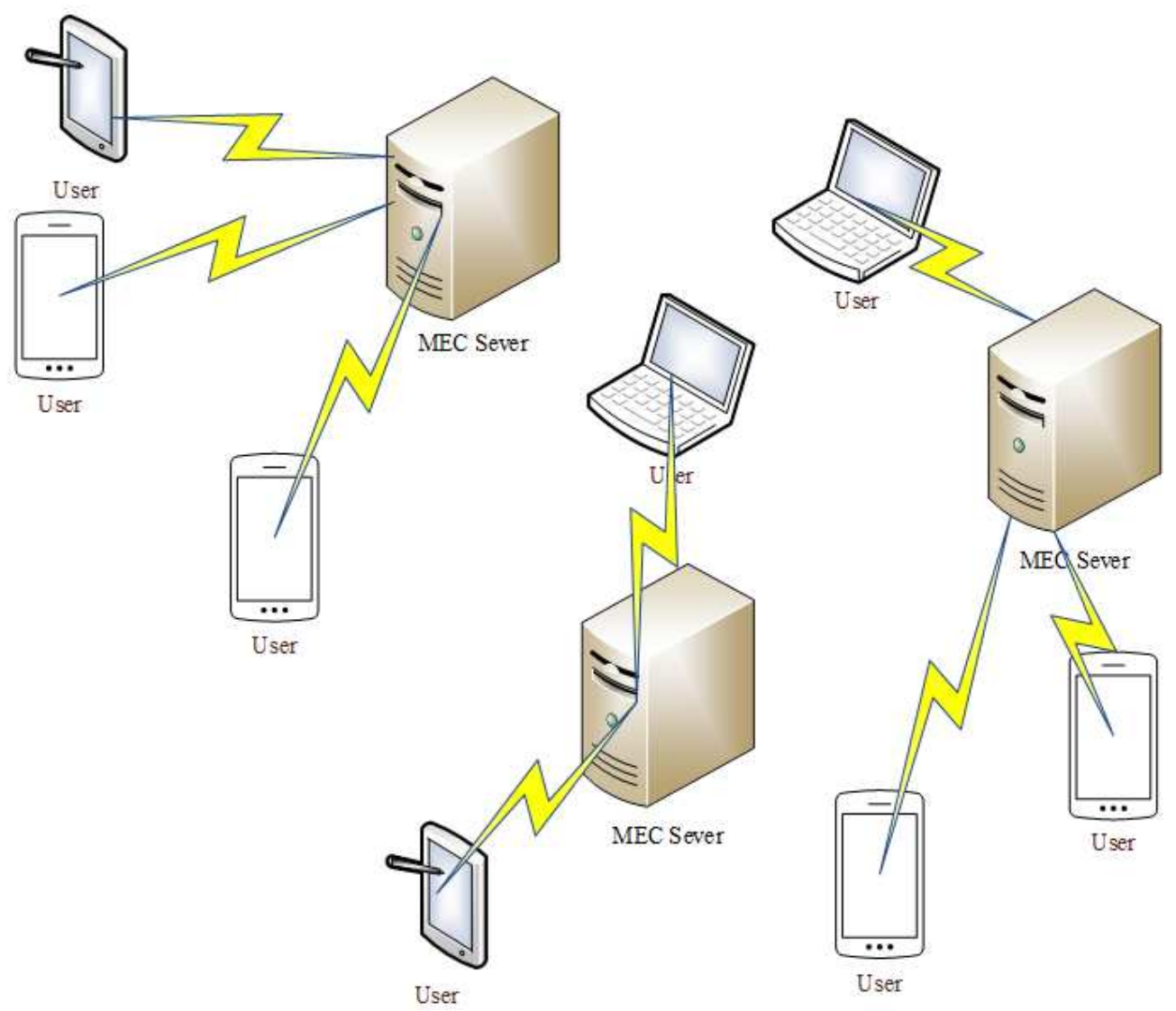

Figure 2. MEC scenario.

The purpose of this paper is to find an offloading strategy that can balance and minimize the average execution delay of tasks and the average energy consumption of device. Combined with the multi-objective optimization algorithm SPEA2, the Pareto front meeting the requirements is obtained. The Pareto front obtained from the experiment is shown in Figure 3.

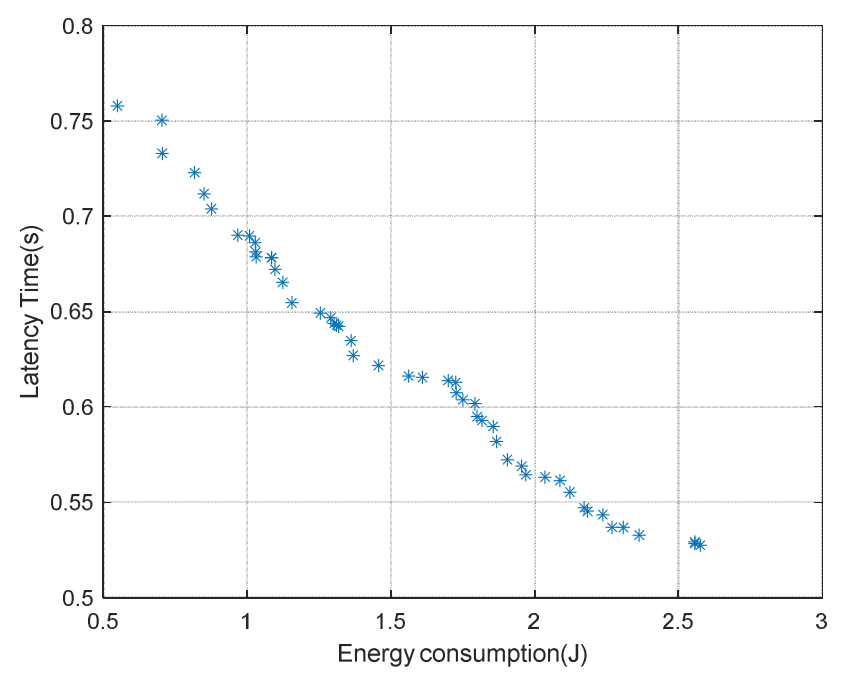

Although Pareto front meets the requirements, we still need to find out the compromise optimal solution to meet the needs of users. Therefore, using combination weighting method, this paper finds out the compromise optimal solution to meet the needs of users from the Pareto front.

The Pareto optimal solution set has two objectives and 50 sets of optimal solutions. Firstly, combine the equation (2) to standardize the optimal solution set of Pareto, to get the normalized matrix $\mathrm{A}=\left(\mathrm{a}_{\mathrm{ij}}\right)_{50 \times 2}$. Then, the subjective weight matrix of the Pareto optimal solution set is obtained by combining analytic hierarchy process. It is assumed that the decision-maker considers the importance of the average equipment execution delay is higher than the task execution energy consumption. Therefore, the decision matrix for the optimal solution set of Pareto is:

$$
\mathrm{O}=\left[\begin{array}{ll}
1 & \frac{1}{3} \\
3 & 1
\end{array}\right]
$$

The results of subjective weighting method and objective weighting method are shown in Table 1 and Table 2 respectively.

Figure 3. Pareto front in MEC scenario. 
Table 1. Analytic hierarchy process results.

\begin{tabular}{lll}
\hline Index & Feature vector & Weight value \\
\hline Energy consumption & 0.500 & 0.25 \\
Latency Time & 1.500 & 0.75 \\
\hline
\end{tabular}

Table 2. Entropy method results.

\begin{tabular}{llll}
\hline Index & $\begin{array}{l}\text { Information } \\
\text { entropy e }\end{array}$ & $\begin{array}{l}\text { Information entropy } \\
\text { redundancy d }\end{array}$ & $\begin{array}{l}\text { Weight } \\
\text { value }\end{array}$ \\
\hline Energy consumption & 0.9959 & 0.0041 & 0.4595 \\
Latency Time & 0.9951 & 0.0049 & 0.5405 \\
\hline
\end{tabular}

From table 1 and table 2, we can know the subjective weight matrix $\mathrm{W}_{\mathrm{u}}=[0.25,0.75]$, and the objective weight matrix $\mathrm{W}_{v}=[0.4595,0.5405]$.

Finally, we use the equation (10) to get the combined weight matrix of the Pareto optimal set through the least square method. The combined weight matrix $\mathrm{W}=[0.364,0.636]$. Use the equation (9) to evaluate these 50 sets of Pareto optimal solutions respectively and get their comprehensive evaluation value $y_{i}$. The solution with the lowest evaluation value is selected as the compromise optimal solution of the Pareto optimal solution set to meet the needs of users in the MEC scenario. Its Pareto front is shown in Figure 4.

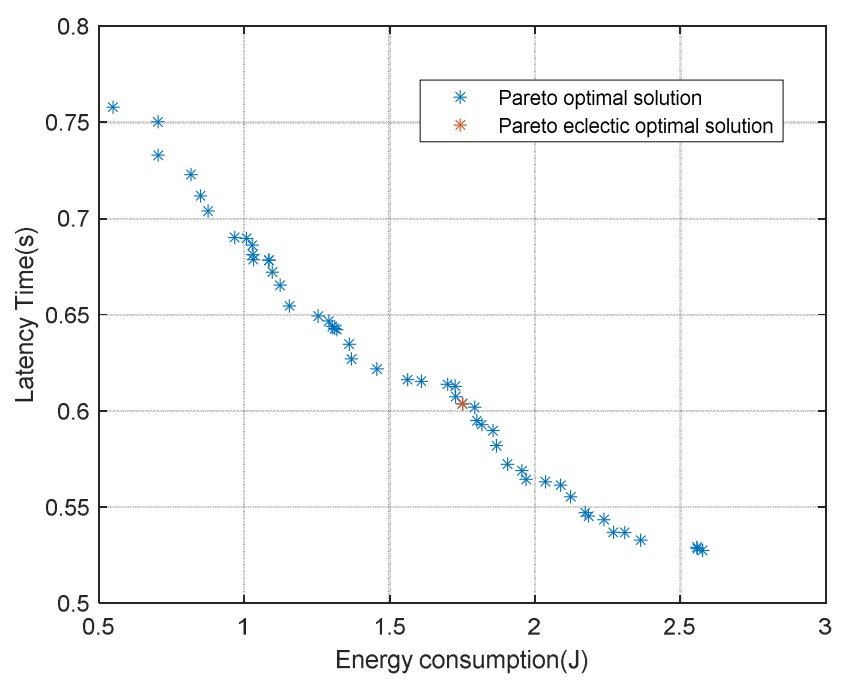

Figure 4. Pareto front with compromise optimal solution.

Generally, we believe that the ideal best point of the multi-objective problem of finding the minimum is the origin. However, as long as the equipment performs task processing, task execution delay and equipment energy consumption will be generated. Therefore, the compromise optimal solution we choose from the Pareto optimal solution can only be as close to the ideal optimal solution as possible.

When users think that task execution delay is more important than equipment energy consumption, the weight of task execution delay is also greater than equipment energy consumption in the obtained combined weight matrix. But at the same time, it also takes into account the objective information of the two indicators, and the weight distribution is more neutral.

The best compromise we finally found was $(1.751,0.6037)$. As shown in Figure 4, even in the whole Pareto front, it is close to the ideal best advantage. This shows that the best compromise selected by the combination weighting method is reasonable.

\section{Conclusion}

This paper is to find an unloading strategy that can not only meet the needs of users, but also meet the objective information of indicators in the Internet of things scenario. This paper combines the combined weighting method in the subjective and objective integrated weighting method, which can finds the compromise optimal solution from the Pareto optimal solution set for the Internet of things scenario. Firstly, through the analytic hierarchy process in the subjective weighting method, the subjective weight matrix which reflects the subjective consciousness of decision-makers is obtained. Then, through the entropy method in the objective weighting method, the objective weight matrix which can reflect the objective information of the index is obtained. Finally, the subjective and objective integrated weight matrix is obtained by combining the least square method. The performance of each group of optimal solutions in the Pareto optimal solution set is evaluated by integrating the weight. And the solution with the lowest comprehensive index is selected as the compromise optimal solution of the Pareto optimal solution set. This compromise optimal solution can not only meet the subjective needs of users in MEC scenario, but also meet the objective information of indicators. In the future, we will further explore the application of the proposed method in the MEC scenario, to find an offloading strategy that can meet the needs of users.

\section{Acknowledgements}

This work was supported in part by National Natural Science Foundation of China (grant no. 61902268), Sichuan Science and Technology Program (grant no. 2018JY0197, 19ZDZX0037, 2019YFSY0045, 20ZDYF0919, 21ZDYF4052, 2020YFH0124, 2021YFSY0060).

\section{References}

[1] Meng, T., Wolter, K., Wu, H., \& Wang, Q. (2018). A secure and cost-efficient offloading policy for mobile cloud computing against timing attacks. Pervasive and Mobile Computing, 45, 4-18. doi: 10.1016/j.pmcj.2018.01.007.

[2] Noor, T. H., Zeadally, S., Alfazi, A., \& Sheng, Q. Z. (2018). Mobile cloud computing: Challenges and future research directions. Journal of Network and Computer Applications, 115, 70-85. doi: 10.1016/j.jnca.2018.04.018.

[3] Ren, J., Zhang, D., He, S., Zhang, Y., \& Li, T. (2019). A survey on end-edge-cloud orchestrated network computing paradigms: Transparent computing, mobile edge computing, fog computing, and cloudlet. ACM Computing Surveys (CSUR), 52 (6), 1-36. doi: 10.1145/3362031. 
[4] McClellan, M., Cervelló-Pastor, C., \& Sallent, S. (2020). Deep learning at the mobile edge: Opportunities for 5G networks. Applied Sciences, 10 (14), 4735. doi: 10.3390/app10144735.

[5] Taha, M. Y., Kurnaz, S., Ibrahim, A. A., Mohammed, A. H., Raheem, S. A., \& Namaa, H. M. (2020, October). Internet Of Things And Cloud Computing-A Review. In 2020 4th International Symposium on Multidisciplinary Studies and Innovative Technologies (ISMSIT) 1-7. doi: 10.1109/ISMSIT50672.2020.9254340.

[6] Ren, J., He, Y., Huang, G., Yu, G., Cai, Y., \& Zhang, Z. (2019). An edge-computing based architecture for mobile augmented reality. IEEE Network, 33 (4), 162-169. doi: 10.1109/MNET.2018.1800132.

[7] Liu, Y., Peng, M., Shou, G., Chen, Y., \& Chen, S. (2020). Toward edge intelligence: Multiaccess edge computing for $5 \mathrm{G}$ and Internet of Things. IEEE Internet of Things Journal, 7 (8), 6722-6747. doi: 10.1109/JIOT.2020.3004500.

[8] Shirazi, S. N., Gouglidis, A., Farshad, A., \& Hutchison, D. (2017). The extended cloud: Review and analysis of mobile edge computing and fog from a security and resilience perspective. IEEE Journal on Selected Areas in Communications, 35 (11), 2586-2595. doi: 10.1109/JSAC.2017.2760478

[9] Shahzadi, S., Iqbal, M., Dagiuklas, T., \& Qayyum, Z. U. (2017). Multi-access edge computing: open issues, challenges and future perspectives. Journal of Cloud Computing, 6 (1), 1-13. doi: 10.1186/s13677-017-0097-9.

[10] Abbas, N., Zhang, Y., Taherkordi, A., \& Skeie, T. (2017). Mobile edge computing: A survey. IEEE Internet of Things Journal, 5 (1), 450-465. doi: 10.1109/JIOT.2017.2750180.

[11] Cui, L., Xu, C., Yang, S., Huang, J. Z., Li, J., Wang, X.,... \& $\mathrm{Lu}, \mathrm{N}$. (2018). Joint optimization of energy consumption and latency in mobile edge computing for Internet of Things. IEEE Internet of Things Journal, 6 (3), 4791-4803. doi: 10.1109/JIOT.2018.2869226.

[12] Ngatchou, P., Zarei, A., \& El-Sharkawi, A. (2005). Pareto multi objective optimization. In Proceedings of the 13th International Conference on, Intelligent Systems Application to Power Systems. IEEE. 84-91 doi: 10.1109/ISAP.2005.1599245.

[13] Vaidya, O. S., \& Kumar, S. (2006). Analytic hierarchy process: An overview of applications. European Journal of operational research, 169 (1), 1-29. doi: 10.1016/j.ejor.2004.04.028.

[14] Botev, Z. I., Kroese, D. P., Rubinstein, R. Y., \& L'Ecuyer, P. (2013). The cross-entropy method for optimization. In Handbook of statistics, 31, 35-59. doi: 10.1016/B978-0-444-53859-8.00003-5.

[15] Li, J., Fang, H., \& Song, W. (2019). Sustainable supplier selection based on SSCM practices: A rough cloud TOPSIS approach. Journal of cleaner production, 222, 606-621. doi: 10.1016/j.jclepro.2019.03.070. 